\title{
DETECTION AND EVALUATION OF HUMAN AND MACHINE GENERATED SPEECH IN SPOOFING ATTACKS ON AUTOMATIC SPEAKER VERIFICATION SYSTEMS
}

\author{
Yang Gao*, Jiachen Lian*, Bhiksha Raj ${ }^{+}$, Rita Singh ${ }^{+}$ \\ ${ }^{*}$ Electrical and Computer Engineering, ${ }^{+}$Language Technologies Institute \\ Carnegie Mellon University \\ Pittsburgh, PA, USA - 15213
}

\begin{abstract}
Automatic speaker verification (ASV) systems utilize the biometric information in human speech to verify the speaker's identity. The techniques used for performing speaker verification are often vulnerable to malicious attacks that attempt to induce the ASV system to return wrong results, allowing an impostor to bypass the system and gain access. Attackers use a multitude of spoofing techniques for this, such as voice conversion, audio replay, speech synthesis, etc. In recent years, easily available tools to generate deepfaked audio have increased the potential threat to ASV systems. In this paper, we compare the potential of human impersonation (voice disguise) based attacks with attacks based on machinegenerated speech, on black-box and white-box ASV systems. We also study countermeasures by using features that capture the unique aspects of human speech production, under the hypothesis that machines cannot emulate many of the finelevel intricacies of the human speech production mechanism. We show that fundamental frequency sequence-related entropy, spectral envelope, and aperiodic parameters are promising candidates for robust detection of deepfaked speech generated by unknown methods.
\end{abstract}

Index Terms - Impersonation, Deepfakes, ASV, Spoof detection

\section{INTRODUCTION}

Automatic speaker verification (ASV) systems utilize the biometric information in human speech to verify the identity of a speaker by matching it with the information present in a database (which is also derived from speech samples). Such systems are also vulnerable to malicious attacks where the attacker tries to provide fake biometric information to fool the ASV systems. There are many spoofing methods in use by attackers nowadays, including direct human impersonation of the target, machine assisted-speech generation such as voice conversion (VC), customized and manipulated text-to-speech synthesis (TTS) system outputs, etc. With the advancement of deep learning techniques, especially with advancements in generative models such as generative adversarial networks [1] and wavenet models [2,3], the quality of synthetic speech is getting much closer to natural speech [3]. Attacks carried out using synthetic speech generated by these methods pose serious threats to ASV systems. As a first step, in this paper, we compare the threats from impersonation attacks with the synthetic speech attacks and establish that deep synthesized fakes are in fact the most dangerous attacks for ASV systems.

It is therefore important to be able to distinguish between fake/synthesized and human-generated speech. This is the broader goal of our paper. However, we do not focus on mere feature selections as [4-6], which would be influenced by the dataset choices, models and training procedures. Instead, we start with the hypothesis that machine-generated speech is too consistent in many respects, and machines are unable to emulate the finer level variations found in naturally produced speech signals. In other words, because of the complexity of the human speech production mechanism, human speech has a greater degree of inconsistency than machine-generated speech. We devise experiments to investigate a select set of features that we believe capture some intricacies of humangenerated speech in a manner that machines cannot. To verify the correctness of our hypothesis, we must not only evaluate the features directly on detecting fake and real speech signals but also establish this through applications such as ASV systems, that must then, by the use of these features in the countermeasures, become more impervious to malicious attacks. We propose several speech-generation-related features and verify them to be effective in improving the overall performance of the detection model and ASV systems. The experiments diagram of this paper is shown as in Fig. 1.

\subsection{Prior work}

A number of approaches of varying success have been proposed in the literature to detect fake speech to increase the security of ASV systems against spoofing attacks. For example, the long-running ASVspoof challenge [7.-9] has raised wide efforts in fake speech spoofing attack countermeasures on ASV systems. The main focus of the challenge, however, has been to rank spoof detection countermeasures, and not to carry out an in-depth evaluation of the ASV systems' per- 


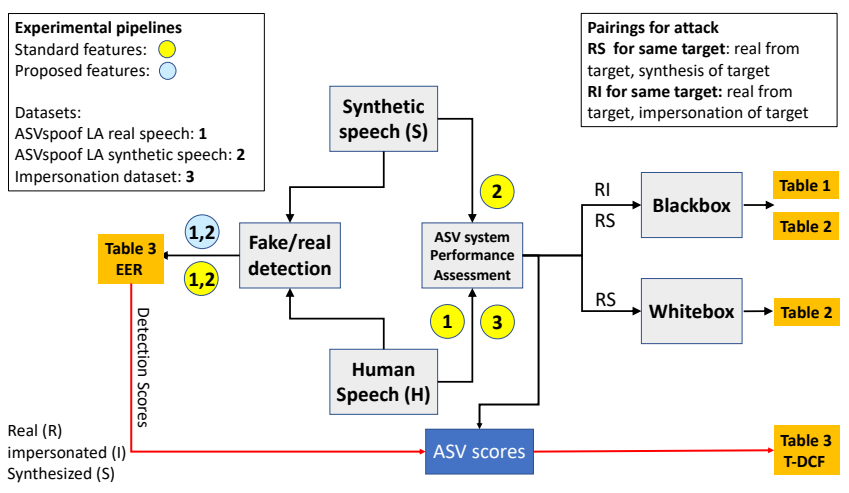

Fig. 1. Diagram for the experimental setups

formance under attacks. Another significant problem with the spoof detection study is that, with the rapid evolution of deepfake generation methodologies, the sheer variety of attacks that an AVS system may be subject to is also rapidly increasing. Detection models trained on a specific provided dataset synthesized using a limited set of methods are not likely to generalize well to newer types of fake/generated audio [10]. In ASVspoof2019, for example, detection algorithms [11-14] that work very well on training datasets are often found to perform much worse on evaluation sets that have been produced using attack techniques not present in the training data. The detection performance on the evaluation sets could be an indicator of the generalization capacity of those proposed algorithms as one purpose of the challenge.

To include the latest deep-learning speech synthesizers, [15] provides a synthetic speech dataset called Fake or Real (FoR), improving variety of the deepfake speech data for this purpose. We have, in fact, also used it effectively in the context of this work.

\section{COMPARING HUMAN AND MACHINE GENERATED SPEECH IN SPOOFING ATTACKS}

As a first step, in this paper, we compare the threats from impersonation attacks with the synthetic speech attacks and establish that deep synthesized fakes are in fact the most dangerous attacks for ASV systems.

\subsection{Datasets}

For our experiments we use four datasets: the logical access (LA) of ASVspoof 2019 dataset [9], the VoxCeleb dataset [16], the FoR dataset [15] and our own collected impersonation dataset (CID).

The CID dataset is collected from the performances of expert impersonators on YouTube, and segmented carefully to only keep the speech segments corresponding to target speakers. All impersonators in the CID dataset are professionals mimicking political figures for amusement, collecting from TV shows and talk shows on YouTube. The dataset comprises 1091 utterances of genuine speech from both the impersonators and the political figures and 981 utterances of impersonated speech. The data are further segregated into paired sets, each pair containing an impersonator's real speech and target/mimicked speech, or the target's real speech and the speech produced for the same target by an impersonator. These are indicated in Table 1, in which pairs that originate from the same speaker are called positive pairs, and pairs from different speakers are called negative pairs.

As shown in Table 1. we have two sets of positive pairs and four sets of negative pairs. They are 19086 positive pairs of same target speaker's real utterances (R), 14844 negative pairs of different target speaker's real utterances (RI), 3382 positive pairs of same impersonator's impersonations for different target (IAB), 37554 negative pairs of target and impersonation pair (TI), 1988 negative pairs of different impersonator's real utterances (IRAB) and 28080 negative pairs of target/impersonator's real utterance pair (IRT).

For synthetic data, ASVspoof2019 dataset contains logical access data and physical access data. In this study, we only use the logical access data which contains machine-generated speech using multiple text-to-speech synthesis and voice conversion methods. The logical data has 2580 bonafide utterances and 22800 synthetic utterances from 20 speakers in the training set; 2548 bonafide utterances from 20 speakers and 22296 spoof utterances from 10 speakers in the development set [9]. The evaluation set contains 7355 bonafide utterances from 67 speakers and 63882 spoof utterances from 48 speakers. The spoof audios are generated using unseen spoofing algorithms intentionally, aiming to give insights of the generalization performance of the proposed countermeasure models. In order for a general ASV system to evaluate this dataset, we generate 4914 bonafide positive pairs and 4914 negative pairs for each attack (A07-A19) from original evaluation set. We also generate 15970 positive pairs and 15970 negative pairs to evaluate the overall attacking ability over all attacks.

To best evaluate the threats of different attacks, we train ASV systems under unconstrained recording and speaking conditions (essentially data-in-the-wild). For this, we use the VoxCeleb dataset, which is a large scale publicly dataset containing millions of utterances collected from unconstrained speech samples [16]. It has many speakers and millions of utterances under different recording conditions. This can be effectively used to evaluate the potential of any given ASV methodology to generalize to unseen speakers and unconstrained conditions [16, 17].

\subsection{Analyzing performance under attacks on black-box and white-box ASV systems}

The ASV model we use is proposed by Joon Son Chung, et al. [17], which applies the Thin ResNet-34 [16] as backbone, and Self-attentive Pooling(SAP) [18] as aggregation strategy. This model, when trained with short-time Fourier transform 
(STFT) spectrogram of Voxceleb, generalizes extremely well to unconstrained conditions as shown by the low EER of real utterance pairs, mentioned earlier in this section.

The black-box ASV system is pretrained with the VoxCeleb dataset. STFT, mel-frequency cepstral coefficients (MFCCs), aperiodic parameters(AP) and spectral envelope(SP) are used as input features to this model. The original input audios comprise segments of $2 \mathrm{sec}$ duration. We use the same STFT feature as in [17] [16]. MFCC feature is computed from $16 \mathrm{kHz}$ sampled signals: which comprise 13 cepstral coefficients, to which first and second-order derivatives respectively are concatenated, making the feature dimensionality 39. (AP and SP are not the focused of this section and will be further discussed in Section 3 and Section 4)

The white-box model is trained with the ASVspoof 2019 data, as a multi-class classifier for speaker identification. We make small modification on the initial ASVspoof2019 training set by assigning each spoofed utterance an identity which uniquely incorporates both speaker and attack. There are 20 speakers and 6 types of attack in the ASVspoof2019 LA training set, meaning that there are 120 "spoofed identities". Thus our modified training set contains 140 identities. We call these ASVspoof training identities (ASVTIs).

\subsubsection{Impersonation attacks}

Our black-box evaluations on impersonation attacks use the CID dataset. We run several experiments to evaluate the dataset's attacking potential. The results are shown in Table 1. The model that is pretrained on VoxCeleb2 is able to verify open-set speakers best and gives $1.71 \%$ EER for target speakers' real utterances (positive and negative pairs $\mathrm{R}+\mathrm{RI}$ ); This pretrained model can be seen as a black box ASV under open-set evaluation.

From our tests, we observe that combining the impersonation/target pairs (TI) with the positive pairs from real speakers $(\mathrm{R})$ improves the speaker verification EER to $11.42 \%$, which indicates that professional impersonation can fool the ASV system to a certain extent, although it is still ineffective in most attacks. The group with real speaker positive pairs (R) and negative pairs (IRAB) built from the real voices of different impersonators has a low EER of $4.86 \%$, showing that the pre-trained ASV system is indeed generalized to verify unseen target speakers and cross impersonators pairs.

The IAB is the same impersonator mimicking different targets. IAB + RI has an EER of $13.3 \%$, showing that even if the same speaker tries to impersonate different targets, their utterances are mostly considered as the same speaker, although still having some capacity to fool the ASV system. Note that this EER value gets significantly larger to $22.09 \%$ with less generalized models, such as the VoxCeleb1 pre-trained model. This indicates that impersonation from professional impersonators is still threatening to some ASV systems. The IAB + IRAB set has comparable EERs as the (a)

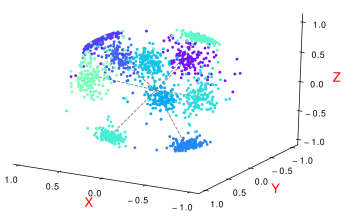

(c)

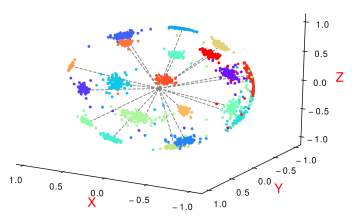

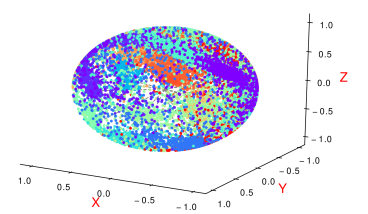

(b)

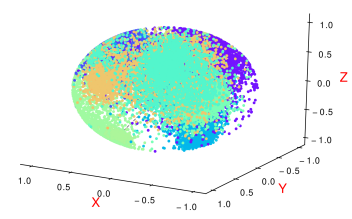

(d)
Fig. 2. (a) 20 bonafide speakers in training set; (b) 20 bonafide and spoof speakers in training set; (c) 10 bonafide speakers in development set; (d) 10 bonafide and spoof speakers in development set.

IAB + RI, showing that the IRAB pairs are valid, also indicating the true differences between the impersonator's real voices. And the IAB + TI gives an EER of $43.52 \%$. This high EER comes from the formation of this evaluation set. Different from other sets, both the negative pairs and positive pairs can be seen as the spoofing attacks because the professional impersonator could impersonate different target to a certain extent, which makes the 'positive' pairs negative in nature. Therefore, both the positive pairs of IAB and the negative pairs of TI are hardest cases, also showing by the EERs of their combination with the $\mathrm{R}$ and $\mathrm{RI}$.

The $\mathrm{R}+$ IRT corresponds to positive pairs for the real voice utterances of the same targets and negative pairs of impersonator's real voice with the targets' real voice. The $5.21 \%$ EER shows that the impersonator's real voices are indeed not similar to the targets' voices.

The overall results show that while mimicry from amateur impersonators is reported to not succeed in fooling ASV systems in previous research [19,20], mimicries rendered by professional impersonators still poses threats to a certain extent.

\subsubsection{Synthetic speech attacks}

To further understand the attacks of synthetic speech generated from different methods, we perform extensive ASV evaluations on the ASVspoof evaluation set under the black-box and white-box conditions. The evaluation set contains attack methods from A07 to A19 which are different voice conversion or speech synthesis techniques [21].

Comparing human-generated attacks and machine generated attacks, as in the black-box scenarios for both cases, we found that the general attack ability of the machine gener- 
Table 1. EERs of impersonation attacks to the ASV under black-box scenario

\begin{tabular}{|c|c|c|c|c|c|c|c|c|c|}
\hline & & \multicolumn{8}{|c|}{ ASV EER\% } \\
\hline \multicolumn{2}{|c|}{ Impersonation Data } & $\mathrm{R}^{1}+\mathrm{RI}^{2}$ & $\mathrm{IAB}+\mathrm{RI}$ & $\mathrm{R}+\mathrm{TI}^{4}$ & $\mathrm{IAB}+\mathrm{TI}$ & $\mathrm{R}+\mathrm{IRAB}^{5}$ & IAB+IRAB & $\mathrm{R}^{\mathrm{IRT}} \mathrm{IR}^{6}$ & IAB+IRT \\
\hline \multirow{5}{*}{ blackbox } & VoxCeleb2(STFT) & 1.71 & 13.30 & 11.42 & 43.52 & 4.86 & 17.76 & 5.21 & 19.45 \\
\hline & VoxCeleb1(STFT) & 4.16 & 16.41 & 14.95 & 42.02 & 4.74 & 15.90 & 5.06 & 15.64 \\
\hline & VoxCeleb1(MFCC) & 17.21 & 22.09 & 22.01 & 48.77 & 9.22 & 26.80 & 8.86 & 20.11 \\
\hline & VoxCeleb1(AP) & 39.75 & 41.27 & 44.89 & 45.46 & 45.58 & 46.06 & 41.65 & 42.94 \\
\hline & VoxCeleb1(SP) & 53.58 & 49.42 & 54.36 & 50.36 & 55.04 & 51.11 & 53.76 & 49.43 \\
\hline \multicolumn{10}{|c|}{${ }^{1}$ R: Same target speaker's real utterance pair (+, \#19086) $\quad{ }^{2}$ RI: Different target speaker's real utterance pair (-, \#14844) } \\
\hline \multicolumn{10}{|c|}{${ }^{3}$ IAB: Same impersonator, impersonations for different target pair $(+, \# 3382) \quad{ }^{4}$ TI: Target and impersonation pair (-, \#37554) } \\
\hline \multicolumn{10}{|c|}{${ }^{5}$ IRAB: Different impersonator's real utterance pair (-, \#1988) $\quad{ }^{6}$ IRT: Target and impersonator's real } \\
\hline \multicolumn{10}{|c|}{${ }^{7}$ The model pre-trained with VoxCeleb2 dev set using Spectrogram feature } \\
\hline
\end{tabular}

Table 2. EERs of evaluation set for ASVspoof2019 LA under black-box and white-box scenarios

\begin{tabular}{|c|c|c|c|c|c|c|c|c|c|c|c|c|c|c|c|}
\hline \multicolumn{16}{|c|}{ ASV EER\% } \\
\hline & Attack & A07 & A08 & A09 & A10 & A11 & A12 & A13 & A14 & A15 & A16 & A17 & A18 & A19 & $\mathrm{ALL}^{3}$ \\
\hline \multirow{6}{*}{ blackbox } & VoxCeleb2(STFT) & 34.03 & 23.20 & 5.70 & 48.51 & 37.37 & 43.42 & 23.67 & 40.45 & 43.14 & 50.51 & 4.99 & 7.10 & 11.26 & 21.42 \\
\hline & VoxCeleb1(STFT) & 27.93 & 25.30 & 11.01 & 47.77 & 37.36 & 44.77 & 30.93 & 43.33 & 40.91 & 43.36 & 7.65 & 10.83 & 13.97 & 22.03 \\
\hline & VoxCeleb1(MFCC) & 45.12 & 28.89 & 16.02 & 45.01 & 48.88 & 45.09 & 38.13 & 35.06 & 43.01 & 46.04 & 11.07 & 25.64 & 25.02 & 25.66 \\
\hline & VoxCeleb1(AP) & 39.89 & 24.92 & 31.63 & 38.66 & 21.93 & 43.05 & 30.99 & 28.25 & 42.21 & 45.55 & 31.56 & 31.92 & 39.27 & 35.55 \\
\hline & VoxCeleb1(SP) & 51.47 & 50.73 & 53.51 & 51.46 & 53.97 & 49.52 & 54.51 & 49.92 & 51.39 & 50.66 & 52.71 & 49.18 & 54.97 & 50.60 \\
\hline & Todisco et al., 9 & 59.68 & 40.39 & 8.38 & 57.73 & 59.64 & 46.18 & 46.78 & 64.01 & 58.85 & 64.52 & 3.92 & 7.35 & 14.58 & - \\
\hline \multirow{9}{*}{ whitebox } & ASVSpoof(STFT) $^{1}$ & 2.33 & 2.65 & 3.75 & 47.56 & 40.89 & 47.59 & 37.01 & 29.09 & 35.48 & 4.09 & 12.07 & 28.61 & 1.88 & 22.24 \\
\hline & ASVSpoof(MFCC) & 7.12 & 5.08 & 8.12 & 39.76 & 28.99 & 49.01 & 33.81 & 19.04 & 41.39 & 9.08 & 18.00 & 16.47 & 2.09 & 15.99 \\
\hline & ASVSpoof(AP) & 38.93 & 32.46 & 32.59 & 42.37 & 38.29 & 43.28 & 37.02 & 33.96 & 41.12 & 49.06 & 40.05 & 34.57 & 44.53 & 39.25 \\
\hline & ASVSpoof(SP) & 50.97 & 49.94 & 40.07 & 49.75 & 49.25 & 52.04 & 52.30 & 51.03 & 51.74 & 51.99 & 41.49 & 46.16 & 45.78 & 42.08 \\
\hline & VoxCeleb2+ASVSpoof(STFT) & 1.16 & 2.31 & 0.77 & 43.42 & 27.62 & 41.23 & 15.46 & 34.48 & 36.26 & 6.63 & 1.26 & 5.75 & 0.68 & 11.99 \\
\hline & VoxCeleb1+ASVSpoof(STFT) & 1.21 & 2.63 & 1.75 & 45.85 & 17.40 & 45.69 & 20.84 & 25.85 & 25.41 & 4.66 & 2.24 & 8.24 & 0.73 & 13.35 \\
\hline & VoxCeleb1+ASVSpoof(MFCC) & 4.99 & 4.51 & 1.99 & 37.28 & 19.02 & 45.08 & 33.18 & 15.92 & 33.65 & 6.01 & 11.30 & 11.44 & 2.98 & 14.94 \\
\hline & VoxCeleb1+ASVSpoof(AP) & 22.04 & 17.77 & 28.13 & 33.68 & 37.78 & 37.20 & 19.72 & 6.50 & 33.16 & 44.43 & 33.25 & 32.33 & 41.01 & 32.45 \\
\hline & VoxCeleb1+ASVSpoof(SP) & 50.24 & 44.30 & 40.51 & 49.21 & 48.82 & 50.45 & 48.74 & 48.62 & 49.43 & 50.86 & 33.63 & 42.10 & 38.06 & 36.79 \\
\hline
\end{tabular}

\footnotetext{
${ }^{1}$ The model trained directly with ASVTIs ${ }^{4}$ using Spectrogram feature

${ }^{2}$ The model pre-trained with VoxCeleb1 dev set using Spectrogram(blackbox) and subsequently trained with ASVTIs ${ }^{3}$ Evaluation on general pairs as described in 2.1, indicating overall EER ${ }^{4}$ As defined in 2.2
}

ated speech are stronger than the human-generated attacks, as shown in Table 1 and Table 2 For the blackbox of VoxCeleb2 trained using STFT, most of the synthetic attacks have a EER of over $20 \%$, much higher than the EER of impersonation attacks (IAB).

As is shown in Table 2 A09/A17/A18/A19 are relatively weaker attacks showing lower ASV EER\% in STFT/MFCCbased black-boxes, which is consistent with the results given by [9]. These attacks are generated through waveform generators such as waveform filtering and spectral filtering, which may be simpler methods compared to hard cases using neural vocoders. Most of attacks tend to be more dangerous for MFCC-based black-box than STFT-based black-boxes. Also for STFT and MFCC, EERs for most attacks are lower under the white-box scenario, compared to attacks on black-boxes with the same dataset and feature settings. However, it does not result in much improvement of EER for A10, A12, and A15, which are generated by neural waveform models, indi- cating increased threat from deepfakes.

In conclusion, the feature robustness ranks as 'STFT > MFCC' with finetuning under white-box scenario. This conclusion is consistent with our hypothesis that features that capture information about prosodic nuances are more robust under attacks for ASV systems.

In Figure. 2, we draw the embedding features from the bottleneck layer of the white-box ASV model on a sphere. When spoofed utterances are introduced, it is not easy to discriminate the embeddings anymore, which indicates their threats to the ASV system.

\section{STRATEGY FOR ESTABLISHING THE GOODNESS OF FEATURES FOR FAKE SPEECH DETECTION}

The threatens shown above from the deepfakes indicates the urgent needs for fake speech detection to assist the anti- 
spoofing capacity of ASV systems. To do so, our final goal is to find robust features for detecting fake speech, especially deepfakes. To re-iterate, our hypothesis is that features that capture the fine-level nuances of human speech from a speech-production perspective are likely to be able to effectively help distinguish between real and fake speech. In addition, they are also likely to improve the performance of countermeasures that are used for thwarting ASV spoofing attacks carried out through synthetic speech.

\subsection{Human voice-production based features}

In the production of speech, there are several sources that are either aperiodic or periodic that generate acoustic energy in the vocal tract. The aperiodic sources are aspiration generated at the glottis, friction generated in the vocal tract, and transient bursts from the rapid release of complete constrictions. The periodic source is the vibration of the vocal folds that creates periodic energy at the glottis. Identifying and quantifying these various sources has several applications in speech coding, speech recognition, and speaker recognition [22].

Synthetic utterances generated by deep generative systems lack specific aspects of naturalness. One notable example is that of prosody. While we do have high quality and plain prosody TTS datasets, these are far from perfect. This is likely to make prosody a promising candidate for our work. Prosody is partially represented through variations in the fundamental frequency (F0) of the speech signal. In addition, features that capture prosody variations are the F0 sequence, spectral envelope, and spectral aperiodicity. We evaluate all of these in our work. Our hypothesis is that features that capture the fine-level nuances of human speech from a speech-production perspective are likely to be able to help distinguish between real and fake speech effectively. Besides, they are also likely to improve the performance of countermeasures that are used for thwarting ASV spoofing attacks carried out through synthetic speech. For example, as shown in Fig.3, the spectral envelope information of fake speech lacks natural transition and nuances, consistent with our hypothesis that the synthetic utterances may lack some aspects of naturalness.

In the vocal production process of a human, the fundamental frequency we refer to is the natural frequency of the vibration of the vocal cords. A specific nuance we can leverage is (known from prior literature) that the larynx can be approximated a nonlinear dynamic system, and the vocal folds can be approximated to coupled oscillators that are theoretically capable of an infinite number of different vibration patterns. However, these are persistently in a perturbed state. In vocal acoustics, perturbation typically refers to a deviation from an expected regularity in vocal-fold vibration. No biological system can produce truly periodic oscillations, and some instantaneous fluctuation can always be expected [23]. Features that capture such instant-to-instant perturbations are the
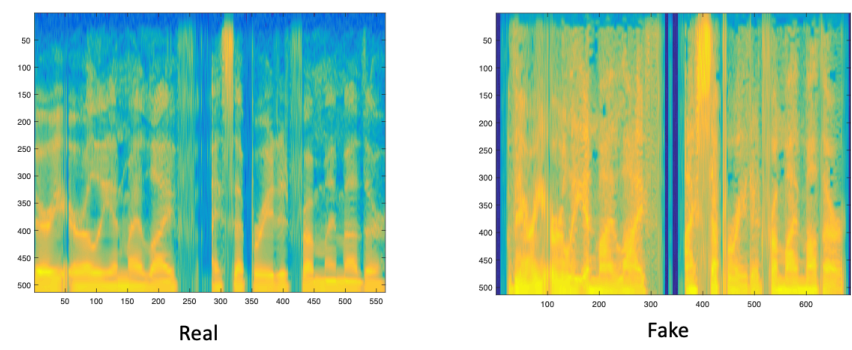

Fig. 3. The same text utterance ("Very early in my life, I separated from my mother." )'s spectral envelope (log scale) of real speech contains natural transition and nuances while the fake speech does not. And the short pause is unnaturally sharp in fake speech.

well-studied jitter and shimmer measurements, which gauge the cycle-to-cycle variations in frequency and amplitude of the speech signal, respectively. It is expected that the information captured by jitter and shimmer may be differently "enacted" in machine-synthesized speech (if at all). We thus choose also to evaluate these features in our work.

\subsection{Analyzing robustness of speech-production moti- vated features}

We are now in a position to analyze the robustness of speech production motivated features for detecting fake speech and improving the robustness of ASV systems against synthetic speech-based attacks. In fact an ASV countermeasure model that evaluates verification performance (based on t-DCF [24] and EER measurement) automatically consolidates and verifies both goals. For reasons explained earlier, we choose to use jitter, shimmer, and other features that capture F0 variations.

For experiments with jitter and shimmer, we only use the utterance-wise average jitter and shimmer values (extracted using Praat [25]), which may not be the best way to use such transient information from speech signals. Nevertheless, we build a three-layer MLP as a countermeasure model that uses these features. In our implementation, we set the F0 range to be within $75-500 \mathrm{~Hz}$. The results show a $31 \%$ EER on the development set, showing that even simple aggregates of these features (the average across an utterance in this case) already make a positive difference to performance.

For experiments with aperiodic and spectral envelope signal features, we verify the spoofing countermeasures in performance improvements. We use the detection model that is modified from the residual net architectures proposed in [11]. To evaluate the proposed features, we do not focus on finetuning parameters and use five residual blocks compared to the 9-11 blocks in [11] for all input features. We set the kernel to be of different sizes to accommodate the dimensionality requirements of the spectral envelope and aperiodic information 
Table 3. ASV countermeasure-based evaluation

\begin{tabular}{c|cc|cc}
\hline & \multicolumn{2}{c}{ Countermeasure EER\% } & \multicolumn{2}{c}{ t_DCF } \\
\hline Features & DEV & EVAL & DEV & EVAL \\
\hline \hline Aperiodic parameters (AP) & 21.19 & 20.65 & 0.4374 & 0.4445 \\
Spectral envelope (SP) & 10.55 & 9.31 & 0.3520 & 0.2453 \\
MFCC & 7.14 & 11.64 & 0.1942 & 0.2663 \\
CQCC & 1.37 & 10.89 & 0.0407 & 0.2746 \\
Spectrogram & 0.48 & 9.39 & 0.0132 & 0.1954 \\
AP+SP & 9.41 & 8.91 & 0.2872 & 0.2462 \\
AP+SP+MFCC & 5.14 & 8.48 & 0.1560 & 0.2169 \\
AP+SP+CQCC & 3.85 & 6.73 & 0.1293 & 0.1777 \\
AP+SP+Spectrogram & 0.62 & 6.67 & 0.0201 & 0.1604 \\
\hline
\end{tabular}

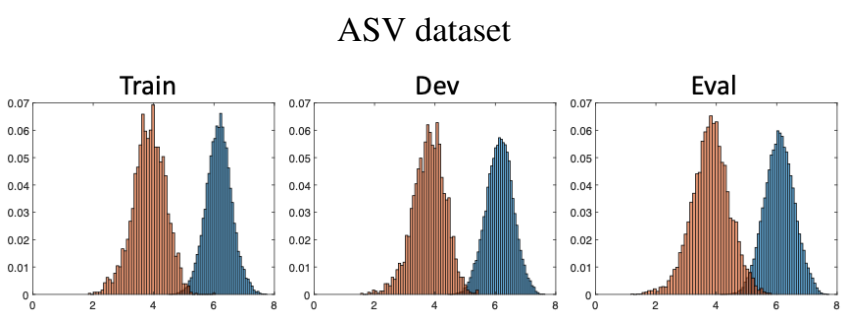

FoR dataset

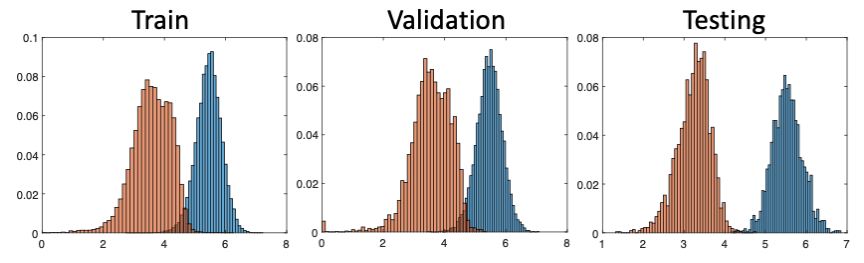

Fig. 4. Spectral entropy distributions. Blue is for fake speech and orange is for real speech

extracted using WORLD [26]. In our evaluation of these features, from Table 3, the EERs are similar for the dev and eval set using these features alone. We can also see that the fusion of aperiodic information and spectral envelope with MFCC or spectrogram or Constant Q cepstral coefficients (CQCC) [27] features can improve the detection performance as evaluated by EER and the joint performance with ASV evaluated by the t-DCF [9.24] and decrease the gap between the EERs of the evaluation set and development set.

In the case of spectral entropy of F0 sequence, our hypothesis is that the F0 sequence of synthetic speech may lack the characteristic shift and variation of natural speech. We use the Shannon entropy of the power spectral density of the F0 sequence to capture this. The equations for the computation of this spectral density are as equations (1), (2) and (3), which first calculate the power spectral density (PSD) of your signal's spectrum $X\left(w_{i}\right)$; then normalize the PSD as probability density function; and finally compute the power spectral entropy.

$$
P\left(w_{i}\right)=\frac{1}{N}\left|X\left(w_{i}\right)\right|^{2}
$$

$$
\begin{gathered}
P_{i}=\frac{P\left(w_{i}\right)}{\sum_{i} P\left(w_{i}\right)} \\
P S E=-\sum^{n} p_{i} \ln p_{i}
\end{gathered}
$$

The F0 sequence is extracted using WORLD [26], and is trimmed to remove the zero values at the beginning and end of the sequence. We plot the spectral entropy distributions for the ASVspoof 2019 logical data's train/dev/eval set and find consistent patterns in them. To evaluate the stability/significance of the patterns, we also compute the distribution from the FoR dataset, as shown in Fig. 4. Results show that the spectral entropy of F0 sequence is a surprisingly good indicator that captures statistical differences between synthetic speech and natural speech across datasets.

\section{DISCUSSIONS}

To further understand the anti-spoofing properties of the aperiodic signal and spectral envelope signals, we evaluated their performance with the direct usage in the ASV model. As shown in Table 1 and Table 2, AP/SP-based blackboxes and white-boxes show much larger ASV EER\% than STFT/MFCC based manners under most attacks. This is even much more obvious in SP-based boxes. The potential speculation is that both AP and SP are the features corresponding to identity-independent attributes like content-dependent attributes. SP is even mostly disentangled from speaker identities. These results are expected since the AP and SP signals are chosen to capture the nuances differences between natural speech and fake speech, while ASV systems requires features that distinguish the speakers' voice characteristics in a finer level. Still, one interesting phenomena we noticed is that AP/SP features, especially AP, seems to be good supplementary information that gives lower EER\% for attacks that STFT/MFCC are not good at. This is consistent with our hypothesis that they could capture the signature information to distinguish human-generated speech and machine-generated speech.

\section{CONCLUSIONS}

In this study, we have established that spoofing attacks carried out using deep-fake speech are more likely to be effective than those using other synthetic methods or human impersonation; even the speech is produced by professional impersonators. We have also established that features that capture the finelevel inconsistencies and nuances of the speech production process could consistently exhibit differences between synthetic speech and genuine speech. All of these result in more robust detection of spoofed speech, and result in rendering ASV systems more robust to attacks generated using unseen methods. 


\section{REFERENCES}

[1] Yang Gao, Rita Singh, and Bhiksha Raj, "Voice impersonation using generative adversarial networks," in 2018 IEEE International Conference on Acoustics, Speech and Signal Processing (ICASSP). IEEE, 2018, pp. 2506-2510.

[2] Aäron van den Oord, Sander Dieleman, Heiga Zen, Karen Simonyan, Oriol Vinyals, Alex Graves, Nal Kalchbrenner, Andrew Senior, and Koray Kavukcuoglu, "WaveNet: A generative model for raw audio," in 9th ISCA Speech Synthesis Workshop, 2016, pp. 125-125.

[3] Jonathan Shen, Ruoming Pang, Ron J Weiss, Mike Schuster, Navdeep Jaitly, Zongheng Yang, Zhifeng Chen, Yu Zhang, Yuxuan Wang, Rj Skerrv-Ryan, et al., "Natural TTS synthesis by conditioning WaveNet on mel spectrogram predictions," in 2018 IEEE International Conference on Acoustics, Speech and Signal Processing (ICASSP). IEEE, 2018, pp. 4779-4783.

[4] BT Balamurali, Kinwah Edward Lin, Simon Lui, JerMing Chen, and Dorien Herremans, "Toward robust audio spoofing detection: A detailed comparison of traditional and learned features," IEEE Access, vol. 7, pp. 84229-84241, 2019.

[5] Madhu R Kamble, Hardik B Sailor, Hemant A Patil, and Haizhou Li, "Advances in anti-spoofing: from the perspective of ASVspoof challenges," APSIPA Transactions on Signal and Information Processing, vol. 9, 2020.

[6] Hong Yu, Zheng-Hua Tan, Yiming Zhang, Zhanyu Ma, and Jun Guo, "DNN filter bank cepstral coefficients for spoofing detection," Ieee Access, vol. 5, pp. 4779-4787, 2017.

[7] Zhizheng Wu, Tomi Kinnunen, Nicholas Evans, Junichi Yamagishi, Cemal Hanilçi, Md Sahidullah, and Aleksandr Sizov, "ASVspoof 2015: the first automatic speaker verification spoofing and countermeasures challenge," in Sixteenth Annual Conference of the International Speech Communication Association, 2015.

[8] Tomi Kinnunen, Md Sahidullah, Héctor Delgado, Massimiliano Todisco, Nicholas Evans, Junichi Yamagishi, and Kong Aik Lee, "The ASVspoof 2017 challenge: Assessing the limits of replay spoofing attack detection," Proc. Interspeech 2017, pp. 2-6, 2017.

[9] Massimiliano Todisco, Xin Wang, Ville Vestman, Md Sahidullah, Héctor Delgado, Andreas Nautsch, Junichi Yamagishi, Nicholas Evans, Tomi Kinnunen, and Kong Aik Lee, "ASVspoof 2019: Future horizons in spoofed and fake audio detection," arXiv preprint arXiv:1904.05441, 2019.
[10] Tianxiang Chen, Avrosh Kumar, Parav Nagarsheth, Ganesh Sivaraman, and Elie Khoury, "Generalization of audio deepfake detection," in Proc. Odyssey 2020 The Speaker and Language Recognition Workshop, 2020, pp. 132-137.

[11] Moustafa Alzantot, Ziqi Wang, and Mani B Srivastava, "Deep residual neural networks for audio spoofing detection," Proc. Interspeech 2019, pp. 1078-1082, 2019.

[12] Rohan Kumar Das, Jichen Yang, and Haizhou Li, "Long range acoustic and deep features perspective on ASV spoof 2019," in IEEE Autom. Speech Recognit. Understanding Workshop, 2019.

[13] Jichen Yang, Rohan Kumar Das, and Haizhou Li, "Significance of subband features for synthetic speech detection," IEEE Transactions on Information Forensics and Security, 2019.

[14] Hossein Zeinali, Themos Stafylakis, Georgia Athanasopoulou, Johan Rohdin, Ioannis Gkinis, Lukáš Burget, and Jan Černocký, "Detecting spoofing attacks using VGG and SincNet: BUT-Omilia submission to asvspoof 2019 challenge," Proc. Interspeech 2019, pp. 10731077, 2019.

[15] R. Reimao and V. Tzerpos, "FoR: A dataset for synthetic speech detection," in 2019 International Conference on Speech Technology and Human-Computer Dialogue (SpeD), Oct 2019, pp. 1-10.

[16] Arsha Nagrani, Joon Son Chung, Weidi Xie, and Andrew Zisserman, "Voxceleb: Large-scale speaker verification in the wild," Computer Speech \& Language, vol. 60, pp. 101027, 2020.

[17] Joon Son Chung, Jaesung Huh, Seongkyu Mun, Minjae Lee, Hee Soo Heo, Soyeon Choe, Chiheon Ham, Sunghwan Jung, Bong-Jin Lee, and Icksang Han, "In defence of metric learning for speaker recognition," arXiv preprint arXiv:2003.11982, 2020.

[18] Weicheng Cai, Jinkun Chen, and Ming Li, "Exploring the encoding layer and loss function in end-to-end speaker and language recognition system," 2018.

[19] Tomi Kinnunen, Rosa González Hautamäki, Ville Vestman, and Md Sahidullah, "Can we use speaker recognition technology to attack itself? enhancing mimicry attacks using automatic target speaker selection," in ICASSP 2019-2019 IEEE International Conference on Acoustics, Speech and Signal Processing (ICASSP). IEEE, 2019, pp. 6146-6150.

[20] Ville Vestman, Tomi Kinnunen, Rosa González Hautamäki, and Md Sahidullah, "Voice mimicry attacks assisted by automatic speaker verification," Computer Speech \& Language, vol. 59, pp. 36-54, 2020. 
[21] Xin Wang, Junichi Yamagishi, Massimiliano Todisco, Hector Delgado, Andreas Nautsch, Nicholas Evans, Md Sahidullah, Ville Vestman, Tomi Kinnunen, Kong Aik Lee, Lauri Juvela, Paavo Alku, Yu-Huai Peng, Hsin-Te Hwang, Yu Tsao, Hsin-Min Wang, Sebastien Le Maguer, Markus Becker, Fergus Henderson, Rob Clark, Yu Zhang, Quan Wang, Ye Jia, Kai Onuma, Koji Mushika, Takashi Kaneda, Yuan Jiang, Li-Juan Liu, Yi-Chiao Wu, Wen-Chin Huang, Tomoki Toda, Kou Tanaka, Hirokazu Kameoka, Ingmar Steiner, Driss Matrouf, Jean-Francois Bonastre, Avashna Govender, Srikanth Ronanki, Jing-Xuan Zhang, and Zhen-Hua Ling, "ASVspoof 2019: A large-scale public database of synthesized, converted and replayed speech," 2019.

[22] Om Deshmukh, Carol Y Espy-Wilson, Ariel Salomon, and Jawahar Singh, "Use of temporal information: Detection of periodicity, aperiodicity, and pitch in speech," IEEE Transactions on Speech and Audio Processing, vol. 13, no. 5, pp. 776-786, 2005.

[23] James A Coan and John JB Allen, Handbook of emotion elicitation and assessment, Oxford university press, 2007.

[24] Tomi Kinnunen, Kong Aik Lee, Hector Delgado, Nicholas Evans, Massimiliano Todisco, Md Sahidullah, Junichi Yamagishi, and Douglas A Reynolds, "t-DCF: a detection cost function for the tandem assessment of spoofing countermeasures and automatic speaker verification," in Proc. Odyssey 2018 The Speaker and Language Recognition Workshop, 2018, pp. 312-319.

[25] David Feinberg, "Parselmouth Praat scripts in python," 2019.

[26] Masanori Morise, Fumiya Yokomori, and Kenji Ozawa, "WORLD: a vocoder-based high-quality speech synthesis system for real-time applications," IEICE TRANSACTIONS on Information and Systems, vol. 99, no. 7, pp. 1877-1884, 2016.

[27] Massimiliano Todisco, Héctor Delgado, and Nicholas Evans, "Constant Q cepstral coefficients: A spoofing countermeasure for automatic speaker verification," Computer Speech \& Language, vol. 45, pp. 516-535, 2017. 